\title{
Glycyrrhizin Suppresses the Growth of Human NSCLC Cell Line HCC827 by Downregulating HMGB 1 Level
}

\author{
Xiaojin Wu, ${ }^{1}$ Weitao Wang ${ }^{D},{ }^{2}$ Yuanyuan Chen, ${ }^{1}$ Xiangqun Liu, ${ }^{3}$ Jindong Wang, \\ Xiaobin Qin, ${ }^{5}$ Dawei Yuan, ${ }^{2}$ Tao Yu, ${ }^{5}$ Guangxia Chen, ${ }^{6}$ Yanyan Mi, ${ }^{7}$ Jie Mou, ${ }^{7}$ Jinpeng Cui, ${ }^{8}$ \\ Ankang $\mathrm{Hu}^{9}{ }^{9}$ Yunxiang $\mathrm{E},{ }^{5}$ and Dongsheng Pei ${ }^{10}{ }^{10}$ \\ ${ }^{1}$ Department of Radiation Oncology, The First People's Hospital of Xuzhou, Xuzhou, Jiangsu 221002, China \\ ${ }^{2}$ Genesis Beijing Co., Ltd., Beijing 100102, China \\ ${ }^{3}$ Department of Respiratory Diseases, The First People's Hospital of Xuzhou, Xuzhou, Jiangsu 221002, China \\ ${ }^{4}$ Department of Chest Surgery, The First People's Hospital of Xuzhou, Xuzhou, Jiangsu 221002, China \\ ${ }^{5}$ Department of Tumor, The First People's Hospital of Xuzhou, Xuzhou, Jiangsu 221002, China \\ ${ }^{6}$ Department of Gastroenterology, The First People's Hospital of Xuzhou, Xuzhou, Jiangsu 221002, China \\ ${ }^{7}$ Department of Pharmacy, Xuzhou Medical College, Xuzhou, Jiangsu 221004, China \\ ${ }^{8}$ Clinical Laboratory of Yantaishan Hospital, No. 91, Jiefang Road, Yantai, Shandong 264001, China \\ ${ }^{9}$ Laboratory Animal Center, Xuzhou Medical College, Xuzhou, Jiangsu 221004, China \\ ${ }^{10}$ Department of Pathology, Xuzhou Medical College, Xuzhou, Jiangsu 221004, China
}

Correspondence should be addressed to Dongsheng Pei; dspei@xzhmu.edu.cn

Received 9 October 2017; Revised 24 November 2017; Accepted 29 November 2017; Published 15 January 2018

Academic Editor: Jialiang Yang

Copyright (C) 2018 Xiaojin Wu et al. This is an open access article distributed under the Creative Commons Attribution License, which permits unrestricted use, distribution, and reproduction in any medium, provided the original work is properly cited.

\begin{abstract}
Lung cancer has very high mortality and glycyrrhizin was found to significantly inhibit the growth of lung cancer cells in vitro and tissues in mice. However, the detailed inhibitory role of glycyrrhizin in the growth of lung cancer is still unclear. In this study, we first found that glycyrrhizin inhibited the growth of lung tumor in PDX mice. And high level of HMGB1 promoted the migration and invasion of lung cancer cells, which was suppressed by glycyrrhizin. Moreover, glycyrrhizin reduced the activity of JAK/STAT signaling pathway, which is the upstream regulator of HMGB1. Therefore, this study revealed a potential mechanism by which glycyrrhizin can inhibit the progression of lung cancer.
\end{abstract}

\section{Introduction}

Lung cancer is highly prevalent worldwide. An estimated 526,510 men and women living in the United States had a history of lung cancer in 2016 [1]. Traditionally, lung cancer can be divided into two types: small cell lung cancer (10-15\%) and non-small cell lung cancer (NSCLC) (85-90\%). The 5year survival for NSCLC (21\%) is usually higher than small cell lung cancer (7\%). However, the 5-year relative survival rate of lung cancer is generally lower (17\%) than other cancer types $[1,2]$. Therefore, it is necessary to design novel drugs for effective treatment of lung cancer.

Glycyrrhizin, a glycoconjugated triterpene, is extracted from the roots of licorice plant, Glycyrrhiza glabra. It was first identified as an antiviral drug [3] and has been subsequently used in the treatment of patients with chronic hepatitis B and hepatitis $C$ due to its anti-inflammatory role $[4,5]$. Recently, glycyrrhizin was found to suppress lung adenocarcinoma A549 cell growth by inducing cancer cell apoptosis through downregulating the activity of thromboxane synthase pathway [6]. Furthermore, the growth of lung tumor tissue in PDX mouse model could be effectively inhibited by combining glycyrrhizin with cisplatin treatment, which showed low toxicity and side effects $[6,7]$. Therefore, glycyrrhizin could be developed as a drug for lung cancer therapy.

High Mobility Group Box 1 (HMGB1) is a conserved nonhistone chromosomal protein that regulates nucleosome formation and gene transcription. HMGB1 could also be 
released into extracellular matrix, where it is recognized as a cytokine based on its role in mediating systemic inflammatory response [8]. Further, HMGB1 is involved in cancer progression and observed in various types of cancers [911]. High level of HMGB1 is always associated with cancer metastasis, suggesting that HMGB1 promotes invasion and metastasis of cancer cells [12-16]. Similarly, HMGB1 can promote the migration and invasion of lung cancer cells and regulate the metastasis of lung cancer $[17,18]$. High serum level of HMGB1 can be a potential clinical biomarker for lung cancer [19-22].

Glycyrrhizin was found to inhibit HMGB1 for diagnosing HMGB1-mediated lethal systemic inflammation [23]. Glycyrrhizin could block the chemoattractant and mitogenic activities of HMGB1 by directly binding to its two HMGbox [24]. Moreover, glycyrrhizin could also regulate the expression of HMGB1 after hepatic ischemia-reperfusion (I/R) injury [25] and induction of traumatic pancreatitis in rats [26]. Moreover, glycyrrhizin can suppress tumor growth by reducing the level or activity of HMGB1 [27, 28]. However, whether the anti-lung cancer effect of glycyrrhizin involves suppression of HMGB1 remains unknown.

In this study, glycyrrhizin was found to suppress the tumor growth of NSCLC in PDX mice. Furthermore, the levels of both HMGB1 and its related JAK/STAT3 signal pathway factors were downregulated by treating PDX mice with glycyrrhizin. These findings indicated that glycyrrhizin may be involved in anticancer therapy of NSCLC through downregulating the level of HMGB1.

\section{Materials and Methods}

2.1. Cell Lines. Human NSCLC cell line HCC827 was purchased from the cell bank of Chinese Academy of Sciences and cultured in RPMI 1640 medium containing 10\% fetal bovine serum (FBS).

2.2. PDX Mice Model. The logarithmic growth phase of HCC827 cells was suspended in cell culture medium and the concentration was adjusted to $1 \times 10^{7} \mathrm{ml}^{-1}$. Then, $0.2 \mathrm{ml}$ cell suspension was subcutaneously injected into the back of nude mice, and the tumor formation was visually observed after $5 \mathrm{~d}$. When the tumor grew to a diameter of 5-7 mm, 30 mice were randomly divided into the model group and glycyrrhizic acid treatment group, with 15 rats/group.

2.3. Glycyrrhizin Treatment. Glycyrrhizic acid (purchased from Dalian Meilen Pharmaceutical Technology Development Co., Ltd.) was diluted in DMSO and intraperitoneally injected at a dose of $100 \mathrm{mg} / \mathrm{kg}$ for two days, followed by continuous administration for two weeks. The model control group was injected with the same amount of DMSO.

2.4. Hematoxylin and Eosin Staining. Lung cancer cells from the glycyrrhizin treated and DMSO treated groups were stained by Hematoxylin and Eosin (H\&E), as previously described [29]. First, samples were treated with distilled water and the nuclei were stained with the alum haematoxylin. Then they differentiated with $0.3 \%$ acid alcohol and were

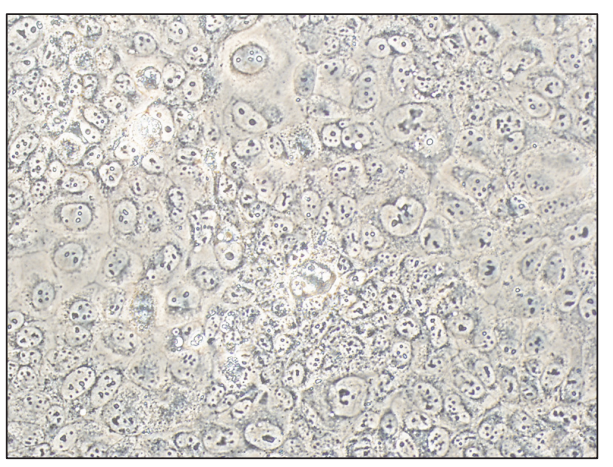

FIGURE 1: Morphology of lung cancer HCC827 cells in PDX mice.

stained with eosin. Finally, they were dehydrated through 95\% alcohol, cleared in 2 changes of xylene, and mounted with xylene based mounting medium.

2.5. Immunohistochemical Staining. The immunohistochemical staining was performed using EnvisionTM, as previously described [30]. Lung cancer tissue staining results were evaluated by IRS score under double-blind conditions by two senior pathological experts. The average staining score was calculated by combining the positive staining intensity and the percentage of positive cells.

2.6. Western Blot Analysis. Western blot analysis was performed as previously described [31]. Cells were lysed using RIPA lysis buffer and then proteins were extracted. Equal amounts of proteins were denatured in boiling SDS sample buffer and subjected to $10 \%$ SDS-PAGE. Then, the proteins were transblotted onto polyvinylidene difluoride membranes with a wet blot system. The membranes were blocked by $5 \%$ dry skim milk and incubated with primary anti-HMGB1 (Abcam), anti-P-Jak2, P-Stat3, Jak2, and Stat3 (Cell Signaling) antibodies. $\beta$-Actin was used as an internal control. Finally, the membranes were treated with enhanced chemiluminescent system for visualization of the protein bands. The bands were quantified using Image J software.

\section{Results}

3.1. Establishment of the PDX Mouse Model for Non-Small Cell Lung Cancer. For testing the effect of glycyrrhizin on the growth of NSCLC, we established the PDX mouse model using the lung cancer HCC827 cell line. Then, we observed the morphology of the cancer cells in PDX mice and found that the arrangement of cells and shape of nuclei were irregular (Figure 1).

3.2. Glycyrrhizin Inhibits Tumor Growth in the PDX Mouse Model. We detected the effect of glycyrrhizin on tumor growth in PDX mice. The PDX mice were divided into two groups: glycyrrhizin treated and DMSO treated. We first observed the morphology of cancer cells by HE staining (Figure 2(a)). Next, we evaluated the tumor sizes and weights in the two groups. The glycyrrhizin treated mice had smaller 

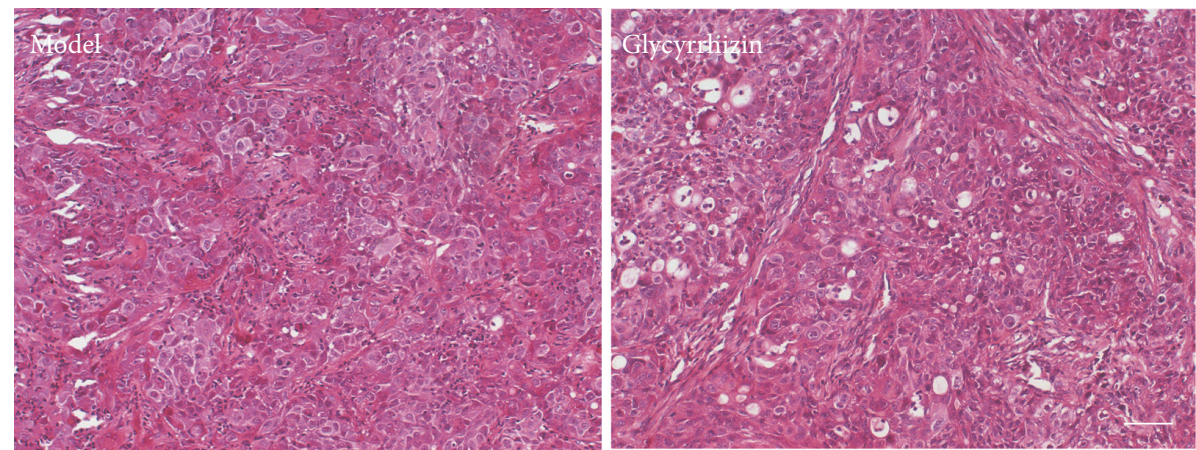

(a)

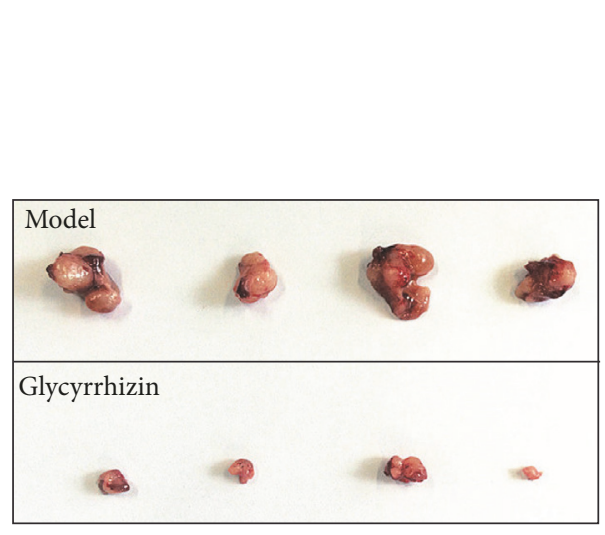

(b)

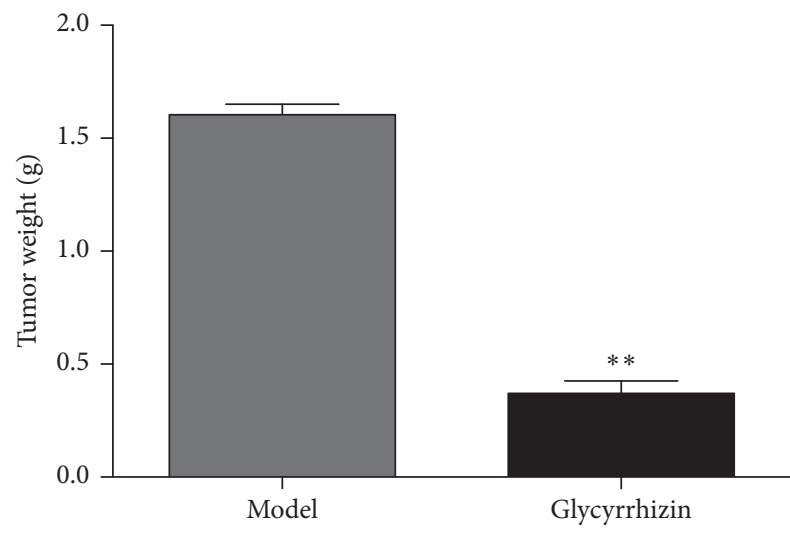

(c)

FIGURE 2: Glycyrrhizin inhibits tumor growth in PDX mice. (a) indicates the morphology of lung cancer cells from the two groups by HE staining, bar $=100 \mu \mathrm{m}$. (b) indicates the size of tumors from the two groups. Tumors were excised from the two groups and the tumor sizes between the two groups were compared. (c) indicates the average weight of tumors in the two groups. The difference of tumor weights between the glycyrrhizin treatment and DMSO treatment (model) was significant. ${ }^{* *} p<0.01$, as compared to the model control by $t$-test.

tumor sizes than the DMSO treated mice (Figure 2(b)). The average tumor weight of the glycyrrhizin treated mice was significantly lower than the DMSO treated group $(p<0.01)$ (Figure 2(c)). These results suggested that glycyrrhizin can inhibit the growth of lung tumor in PDX mice.

\subsection{HMGB1 Protein Is Suppressed by Glycyrrhizin. HMGB1} was reported to promote the migration and invasion of lung cancer cells and facilitate lung cancer metastasis [17, 18]. Glycyrrhizin functions as an inhibitor of HMGB1 by blocking its activity [24] or downregulating its expression [25]. However, whether the anticancer effect of glycyrrhizin in lung cancer relies on downregulating the expression of HMGB1 is unclear. So we detected the protein expression of HMGB1 in lung cancer tissues obtained from the two groups by IHC staining, and representative images are shown in Figure 3(a). Furthermore, the protein level of HMGB1 in the glycyrrhizin treated group was significantly lower than that in the DMSO treated group $(p<0.05$ ) (Figure 3(b)). We also detected the protein level of HMGB1 by western blot and observed similar results. Interestingly, higher level of HMGB1 was also seen in lung tumor tissue from PDX mice compared to that from normal mice (NC), which is consistent with previous reports indicating that HMGB1 is related to cancer progression. However, the level of HMGB1 obviously decreased after glycyrrhizin treatment (Figure 3(c)), suggesting that HMGB1 protein is suppressed by glycyrrhizin.

3.4. Glycyrrhizin Inhibits the Phosphorylation of Jak2 and Stat3. In mammals, the JAK/STAT pathway is the principal signaling mechanism of inflammation and involves various cytokines and growth factors $[32,33]$. Members of the JAK family are receptor-associated tyrosine kinases activated by various extracellular signals. Signal Transducer and Activator of Transcription (STAT) proteins are the typical substrates of JAK kinases and are generally associated with transcriptional activation as transcription factors [34]. HMGB1 is released from the nucleus into the cytoplasm, which is regulated by JAK/STAT signal pathway mediated HMGB1 hyperacetylation [35]. Furthermore, resveratrol could reduce the release of HMGB1 from the nucleus to the cytoplasm by suppressing the activity of STAT signaling pathway [36].

We further examined whether glycyrrhizin could inhibit the activity of JAK/STAT signaling pathway. The phosphorylation status of Jak 2 and Stat 3 was detected by specific phosphorylated antibodies, which showed that the phosphorylation levels of Jak2 and Stat 3 were significantly higher in the PDX-model mice, but obviously lower after glycyrrhizin 


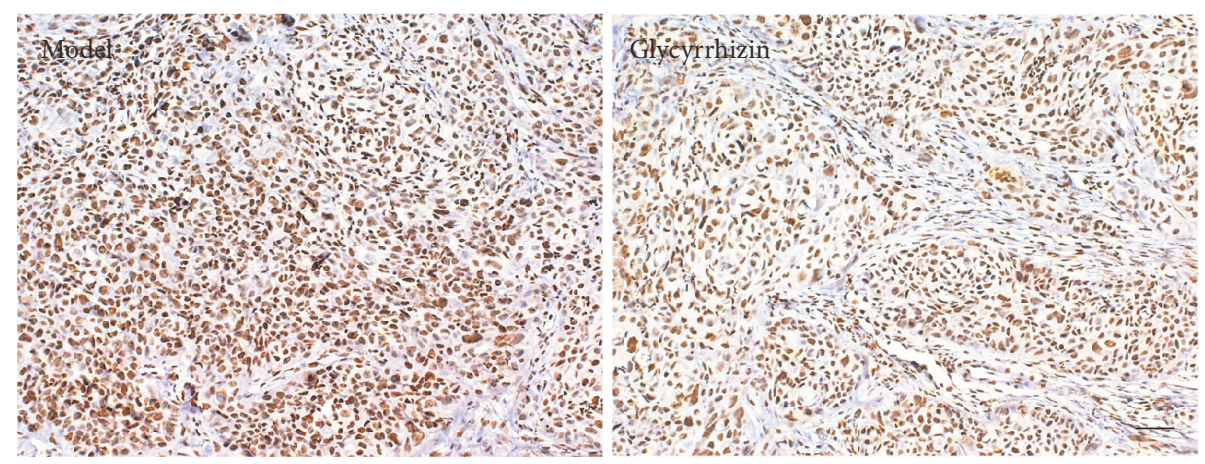

(a)

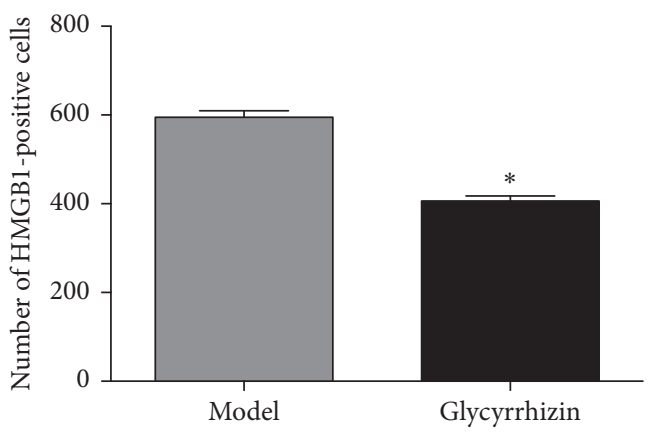

(b)

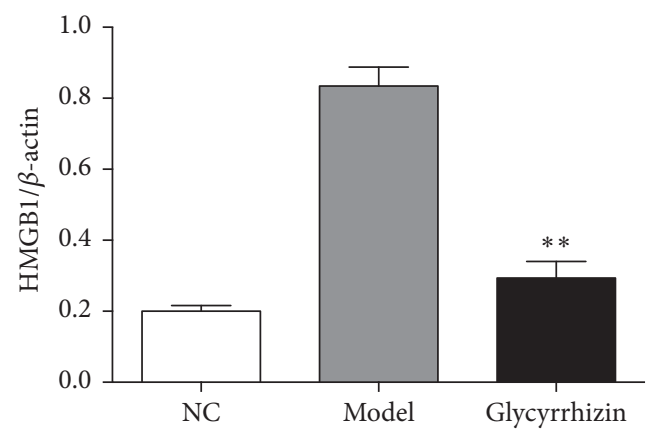

(c)

FIGURE 3: The protein level of HMGB1 is suppressed by glycyrrhizin. (a) indicates the protein level of HMGB1 in lung tumor tissues from the two groups detected by IHC staining assay, bar $=100 \mu \mathrm{m}$. (b) indicates the quantitative results based on IHC staining assay. HMGB1-positive cells were counted in the two groups, respectively. ${ }^{*} p<0.05$, as compared to the model control by $t$-test. (c) indicates the protein level of HMGB1 from the three groups by western blot. $\beta$-Actin was used as an internal control. $p$ value was calculated by $t$-test between glycyrrhizin treatment and DMSO treatment (model). "NC" represents lung tissue from normal mice. ${ }^{* *} p<0.01$, as compared to the model control.

treatment (Figures 4(a) and 4(b)). These results indicated that glycyrrhizin can inhibit the activity of JAK/STAT signaling pathway, which is the upstream regulator of HMGB1.

\section{Discussion}

In this study, glycyrrhizin was shown to suppress the growth of lung tumor tissues in PDX mice, derived from NSCLC HCC827 cell line, which is consistent with recent reports on the anticancer effect of glycyrrhizin on lung cancer progression [6, 7]. Huang et al. [6] showed that glycyrrhizin could inhibit the lung adenocarcinoma A549 cell line growth both in vitro and in PDX mice. Subsequently, Deng et al. [7] reported that glycyrrhizin combined with cisplatin had a better anticancer effect in the PDX mice model. We used another NSCLC cell line for establishing the PDX mice and proved that anticancer effect of glycyrrhizin was similar. Our findings further confirmed that glycyrrhizin may be a potential anticancer drug for NSCLC.

HMGB1, a cytokine, has extracellular functions in inflammation and cancer progression. As a late modulator of inflammation, HMGB1 functions as a damageassociated molecular pattern in the sterile inflammation model by amplifying hepatic ischemia/reperfusion (I/R) and acetaminophen-induced liver necrotic injury [37, 38]. Glycyrrhizin can antagonize the inflammatory effect of HMGB1 by suppressing the expression of HMGB1 in hepatic I/R injury [25]. In addition, HMGB1 has a critical role in cancer metastasis. High levels of HMGB1 are always observed in various cancer types, including ovarian [15], liver [37], and lung [22] cancers. HMGB1 can promote the migration and invasion of lung cancer cells [18]. Drugs designed for regulating the level of HMGB1 may have a potential clinical value for lung cancer patients. In this study, high level of HMGB1 was related to the growth of lung tumors in PDX mice. Glycyrrhizin obviously inhibited the level of HMGB1. Therefore, glycyrrhizin acts as an inhibitor of HMGB1 and the growth of lung tumor. The direct role of HMGB1 in anticancer effect of glycyrrhizin on lung cancer progression needs further study.

Glycyrrhizin influenced the upstream regulator of HMGB1, the JAK/STAT signaling pathway. HMGB1 release from nucleus to cytoplasm is regulated by the activation of JAK/STAT signaling pathway [35]. We found that glycyrrhizin can block the activity of JAK/STAT signaling pathway by inhibiting phosphorylation of Jak2 and Stat3. This is the first study to show that the level of HMGB1 may be controlled by the JAK/STAT signaling pathway.

\section{Conclusion}

Glycyrrhizin inhibits the growth of lung tumors in PDX mice by downregulating the level of HMGB1. This mechanism is 

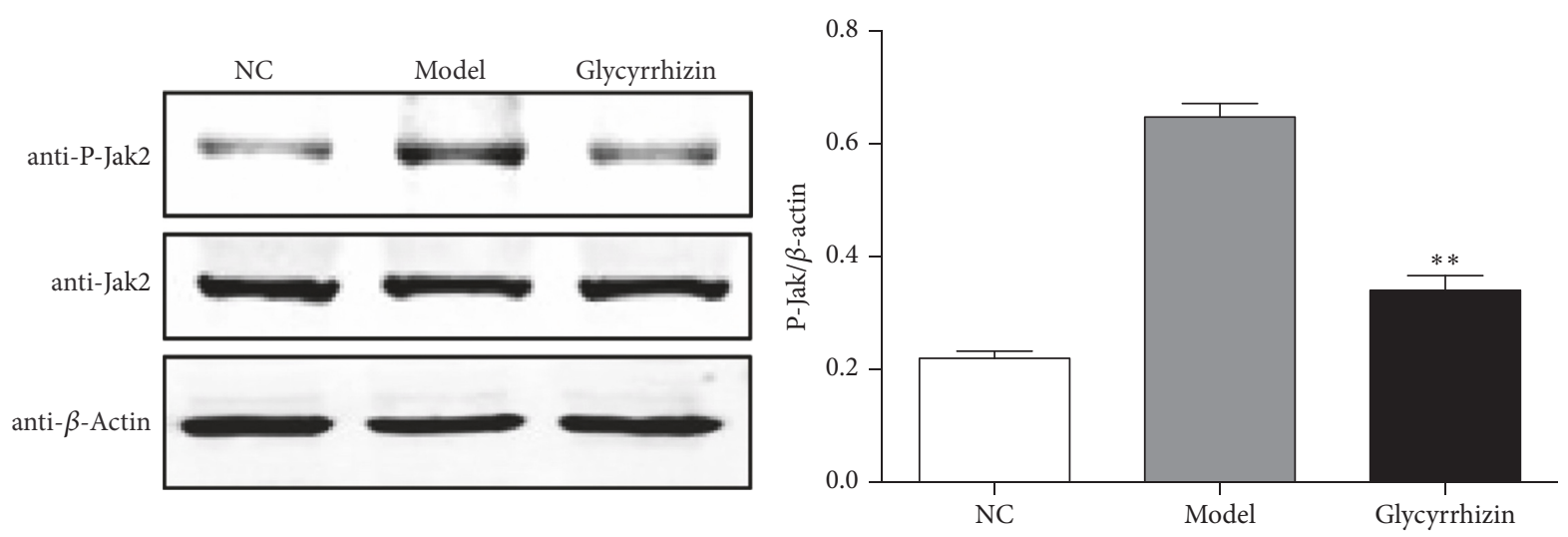

(a)
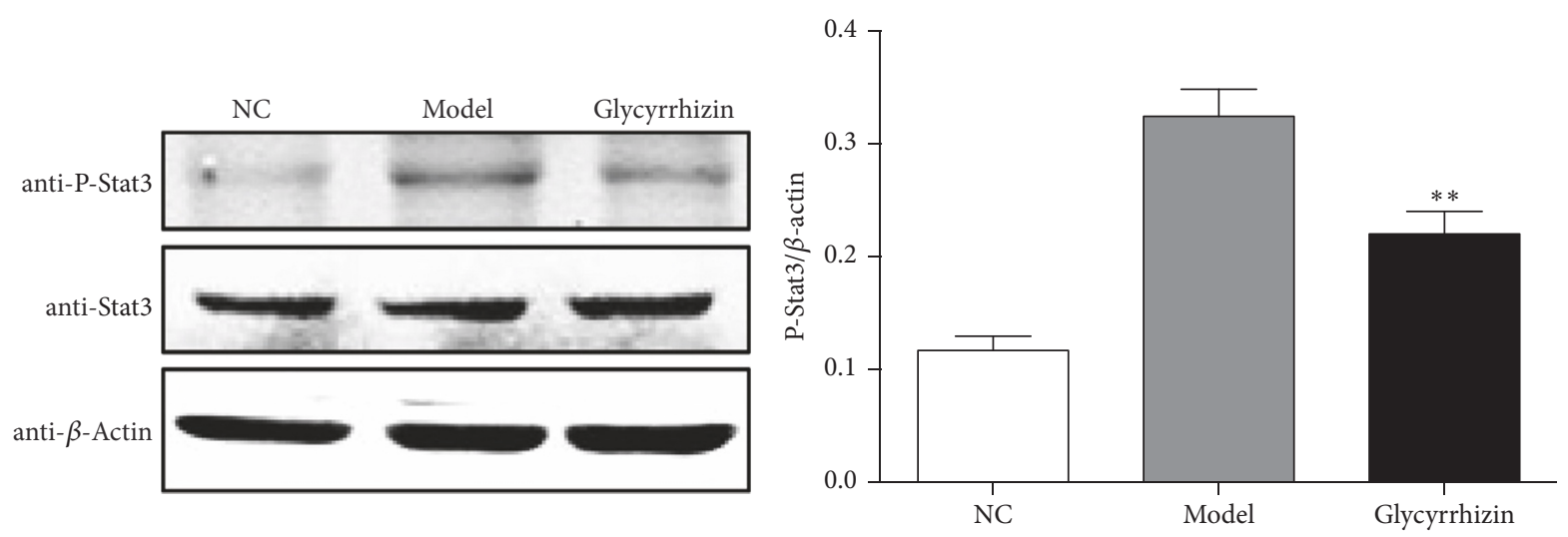

(b)

FIGURE 4: Glycyrrhizin inhibits the phosphorylation of Jak2 and Stat3. The phosphorylation level of Jak2 was detected by western blot in the three experimental groups, respectively. ${ }^{* *} p<0.01$, as compared to the model control (a). The phosphorylation level of Stat 3 was detected by western blot in the three experimental groups, respectively. ${ }^{* *} p<0.01$, as compared to the model control (b). $\beta$-Actin was used as an internal control. $p$ value was calculated by $t$-test between glycyrrhizin treatment and DMSO treatment (model). "NC" represents lung tissue from normal mice. Stars are showing $p$ values between model and glycyrrhizin.

potentially due to the inhibition of JAK/STAT signaling pathway by glycyrrhizin. Glycyrrhizin may be further investigated as a potential drug for NSCLC.

\section{Disclosure}

Xiaojin Wu and Weitao Wang are co-first authors.

\section{Conflicts of Interest}

The authors declare no conflicts of interest.

\section{Acknowledgments}

This study was supported by the Foundation of Jiangsu Provincial Commission of Health and Family Planning (Grant QMRC2016363), Key Talents of Medical Science in Jiangsu Province (Grant Z201627), Xuzhou Administration of Science \& Technology (Grant KC14SH009), and the 333 High-Level Talents of Jiangsu Province (Grant (2016)-1903).

\section{References}

[1] K. D. Miller, R. L. Siegel, and C. C. Lin, "Cancer treatment and survivorship statistics, 2016," CA: A Cancer Journal for Clinicians, vol. 66, no. 4, pp. 271-289, 2016.

[2] S. Subramaniam, R. K. Thakur, V. K. Yadav, R. Nanda, S. Chowdhury, and A. Agrawal, "Lung cancer biomarkers: state of the art," Journal of Carcinogenesis, vol. 12, no. 3, 2013.

[3] R. Pompei, O. Flore, M. A. Marccialis, A. Pani, and B. Loddo, "Glycyrrhizic acid inhibits virus growth and inactivates virus particles," Nature, vol. 281, no. 5733, pp. 689-690, 1979.

[4] S. Iino, T. Tango, T. Matsushima et al., "Therapeutic effects of stronger neo-minophagen $\mathrm{C}$ at different doses on chronic hepatitis and liver cirrhosis," Hepatology Research, vol. 19, no. 1, pp. 31-40, 2001.

[5] K. Miyake, T. Tango, Y. Ota et al., "Efficacy of Stronger NeoMinophagen $\mathrm{C}$ compared between two doses administered three times a week on patients with chronic viral hepatitis," Journal of Gastroenterology and Hepatology, vol. 17, no. 11, pp. 1198-1204, 2002. 
[6] R.-Y. Huang, Y.-L. Chu, Z.-B. Jiang, X.-M. Chen, X. Zhang, and $\mathrm{X}$. Zeng, "Glycyrrhizin suppresses lung adenocarcinoma cell growth through inhibition of thromboxane synthase," Cellular Physiology and Biochemistry, vol. 33, no. 2, pp. 375-388, 2014.

[7] Q.-P. Deng, M.-J. Wang, X. Zeng, G. G. Chen, and R.-Y. Huang, "Effects of glycyrrhizin in a mouse model of lung adenocarcinoma," Cellular Physiology and Biochemistry. International Journal of Experimental Cellular Physiology, Biochemistry and Pharmacology, vol. 41, Article ID 13831392, pp. 1383-1392, 2017.

[8] M. T. Lotze and K. J. Tracey, "High-mobility group box 1 protein (HMGB1): nuclear weapon in the immune arsenal," Nature Reviews Immunology, vol. 5, no. 4, pp. 331-342, 2005.

[9] D. Süren, M. Yildirim, Ö. Demirpençe et al., "The role of High Mobility Group Box 1 (HMGB1) in colorectal cancer," Medical Science Monitor, vol. 20, pp. 530-537, 2014.

[10] Y. R. Choi, H. Kim, H. J. Kang et al., "Overexpression of high mobility group box 1 in gastrointestinal stromal tumors with KIT mutation," Cancer Research, vol. 63, Article ID 21882193, pp. 2188-2193, 2003.

[11] Y. Li, J. Tian, X. Fu et al., "Serum high mobility group box protein 1 as a clinical marker for ovarian cancer," Neoplasma, vol. 61, no. 5, pp. 579-584, 2014.

[12] W. Yan, Y. Chang, X. Liang et al., "High-mobility group box 1 activates caspase- 1 and promotes hepatocellular carcinoma invasiveness and metastases," Hepatology, vol. 55, no. 6, pp. 1863-1875, 2012.

[13] B. Song, W.-G. Song, Z.-J. Li et al., "Effect of HMGB1 silencing on cell proliferation, invasion and apoptosis of MGC-803 gastric cancer cells," Cell Biochemistry \& Function, vol. 30, no. 1, pp. 1117, 2012.

[14] S. Jube, Z. S. Rivera, M. E. Bianchi et al., "Cancer cell secretion of the DAMP protein HMGB1 supports progression in malignant mesothelioma," Cancer Research, vol. 72, no. 13, pp. 3290-3301, 2012.

[15] J. Chen, X. Liu, J. Zhang, and Y. Zhao, "Targeting HMGB1 inhibits ovarian cancer growth and metastasis by lentivirusmediated RNA interference," Journal of Cellular Physiology, vol. 227, no. 11, pp. 3629-3638, 2012.

[16] C. A. Wild, S. Brandau, R. Lotfi et al., "HMGB1 is overexpressed in tumor cells and promotes activity of regulatory $\mathrm{T}$ cells in patients with head and neck cancer," Oral Oncology, vol. 48, no. 5, pp. 409-416, 2012.

[17] C. Zhang, S. Ge, C. Hu, N. Yang, and J. Zhang, "MiRNA218, a new regulator of HMGB1, suppresses cell migration and invasion in non-small cell lung cancer," Acta Biochimica et Biophysica Sinica, vol. 45, Article ID 10551061, pp. 1055-1061, 2013.

[18] C. Wang, G. Fei, Z. Liu, Q. Li, Z. Xu, and T. Ren, "HMGB1 was a pivotal synergistic effecor for $\mathrm{CpG}$ oligonucleotide to enhance the progression of human lung cancer cells," Cancer Biology \& Therapy, vol. 13, no. 9, pp. 727-736, 2012.

[19] G.-H. Shang, C.-Q. Jia, H. Tian et al., "Serum high mobility group box protein 1 as a clinical marker for non-small cell lung cancer," Respiratory Medicine, vol. 103, no. 12, pp. 1949-1953, 2009.

[20] F. Wei, F. Yang, X. Jiang, W. Yu, and X. Ren, "Highmobility group nucleosome-binding protein 1 is a novel clinical biomarker in non-small cell lung cancer," Tumor Biology, vol. 36, no. 12, pp. 9405-9410, 2015.

[21] A. Feng, Z. Tu, and B. Yin, "The effect of HMGB1 on the clinicopathological and prognostic features of non-small cell lung cancer," Oncotarget, vol. 7, pp. 20507-20519, 2016.
[22] Q. Xia, J. Xu, H. Chen et al., "Association between an elevated level of HMGBI and non-small-cell lung cancer: A metaanalysis and literature review," OncoTargets and Therapy, vol. 9, pp. 3917-3923, 2016.

[23] D. Musumeci, G. N. Roviello, and D. Montesarchio, "An overview on HMGB1 inhibitors as potential therapeutic agents in HMGB1-related pathologies," Pharmacology \& Therapeutics, vol. 141, no. 3, pp. 347-357, 2014.

[24] L. Mollica, F. de Marchis, A. Spitaleri et al., "Glycyrrhizin binds to high-mobility group box 1 protein and inhibits its cytokine activities," Chemistry \& Biology, vol. 14, no. 4, pp. 431-441, 2007.

[25] M. Ogiku, H. Kono, M. Hara, M. Tsuchiya, and H. Fujii, "Glycyrrhizin prevents liver injury by inhibition of highmobility group box 1 production by kupffer cells after ischemiareperfusion in rats," The Journal of Pharmacology and Experimental Therapeutics, vol. 339, no. 1, pp. 93-98, 2011.

[26] K. Xiang, L. Cheng, Z. Luo et al., "Glycyrrhizin suppresses the expressions of HMGB1 and relieves the severity of traumatic pancreatitis in rats," PLoS ONE, vol. 9, no. 12, Article ID el15982, 2014.

[27] R. Smolarczyk, T. Cichoń, S. Matuszczak et al., "The role of glycyrrhizin, an inhibitor of HMGB1 protein, in anticancer therapy," Archivum Immunologiae et Therapia Experimentalis, vol. 60, no. 5, pp. 391-399, 2012.

[28] J. F. Curtin, "HMGB1 mediates endogenous TLR2 activation and brain tumor regression," PLOS Medicine, vol. 6, p. e10, 2009.

[29] J. Elsam, "Histological and histochemical methods: theory and practice, $5^{\text {th }}$ edition," Biotechnic \& Histochemistry, vol. 91, no. 2, pp. 145-145, 2016.

[30] E. Sabattini, K. Bisgaard, S. Ascani et al., "The EnVision(TM)+ system: A new immunohistochemical method for diagnostics and research. Critical comparison with the APAAP, ChemMate(TM), CSA, LABC, and SABC techniques," Journal of Clinical Pathology, vol. 51, no. 7, pp. 506-511, 1998.

[31] Y. Zhang, J. Dong, P. He et al., "Genistein inhibit cytokines or growth factor-induced proliferation and transformation phenotype in fibroblast-like synoviocytes of rheumatoid arthritis," Inflammation, vol. 35, no. 1, pp. 377-387, 2012.

[32] J. S. Rawlings, K. M. Rosler, and D. A. Harrison, "The JAK/STAT signaling pathway," Journal of Cell Science, vol. 117, no. 8, pp. 1281-1283, 2004.

[33] C. Schindler, D. E. Levy, and T. Decker, "JAK-STAT signaling: from interferons to cytokines," The Journal of Biological Chemistry, vol. 282, no. 28, pp. 20059-20063, 2007.

[34] D. S. Aaronson and C. M. Horvath, "A road map for those who don't know JAK-STAT,' Science, vol. 296, no. 5573, pp. 16531655, 2002.

[35] B. Lu, D. J. Antoine, K. Kwan et al. et al., "JAK/STAT1 signaling promotes HMGB1 hyperacetylation and nuclear translocation," Proceedings of the National Academy of Sciences of the United States of America, vol. 111, pp. 3068-3073, 2014.

[36] C. Ma, Y. Wang, L. Dong, M. Li, and W. Cai, "Anti-inflammatory effect of resveratrol through the suppression of NF- $\kappa \mathrm{B}$ and JAK/STAT signaling pathways," Acta Biochimica et Biophysica Sinica, vol. 47, pp. 207-213, 2015.

[37] X. Wang, L. Xiang, H. Li et al., “The role of HMGB1 signaling pathway in the development and progression of hepatocellular carcinoma: a review," International Journal of Molecular Sciences, vol. 16, no. 9, pp. 22527-22540, 2015. 
[38] P. Huebener, J.-P. Pradere, C. Hernandez et al., "The HMGB1/ RAGE axis triggers neutrophil-mediated injury amplification following necrosis," The Journal of Clinical Investigation, vol. 125, no. 2, pp. 539-550, 2015. 


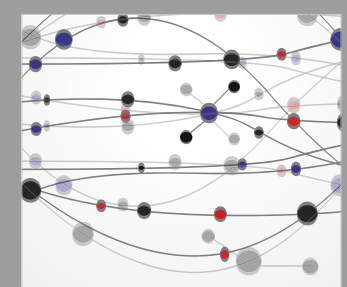

The Scientific World Journal
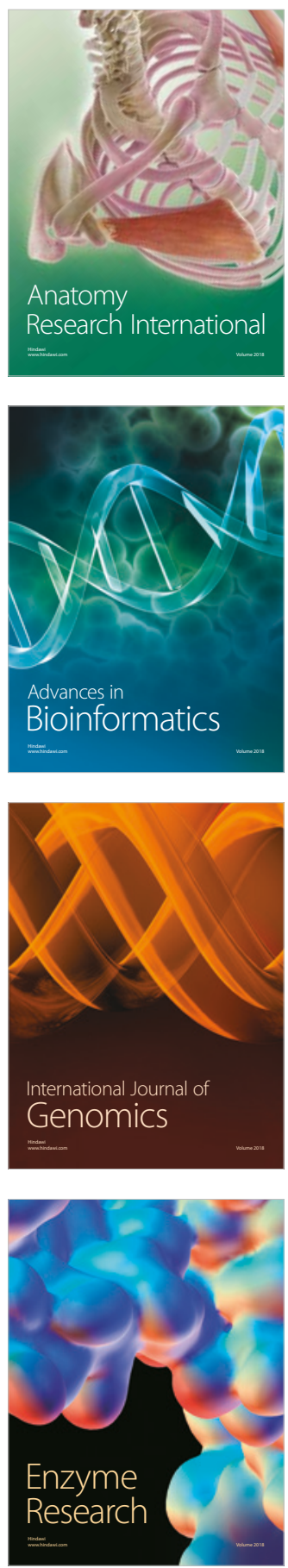
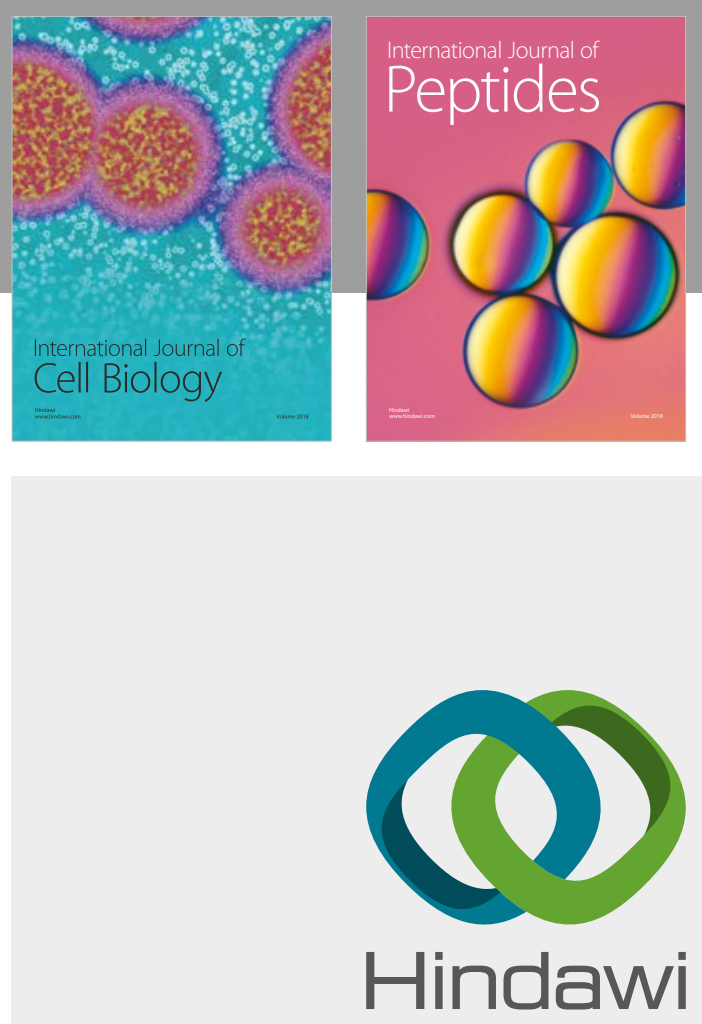

Submit your manuscripts at

www.hindawi.com
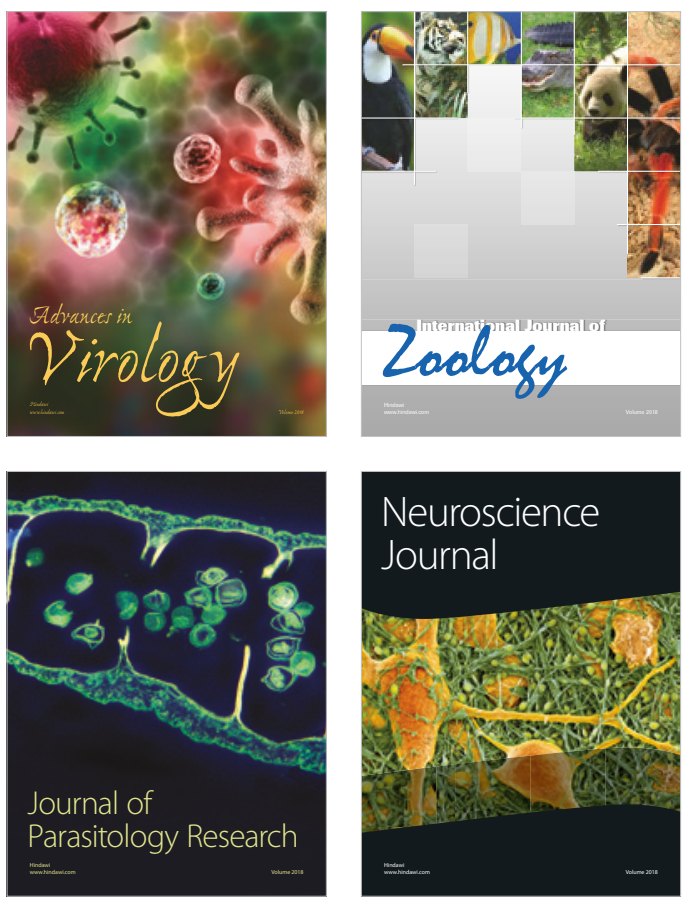
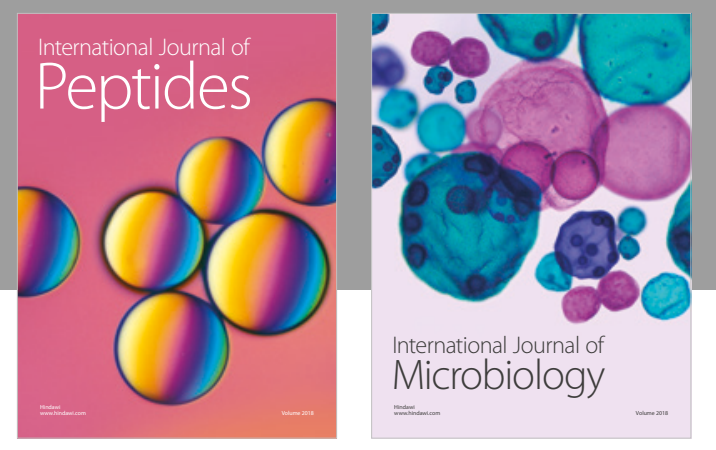

nternational Journal of Microbiology
Journal of
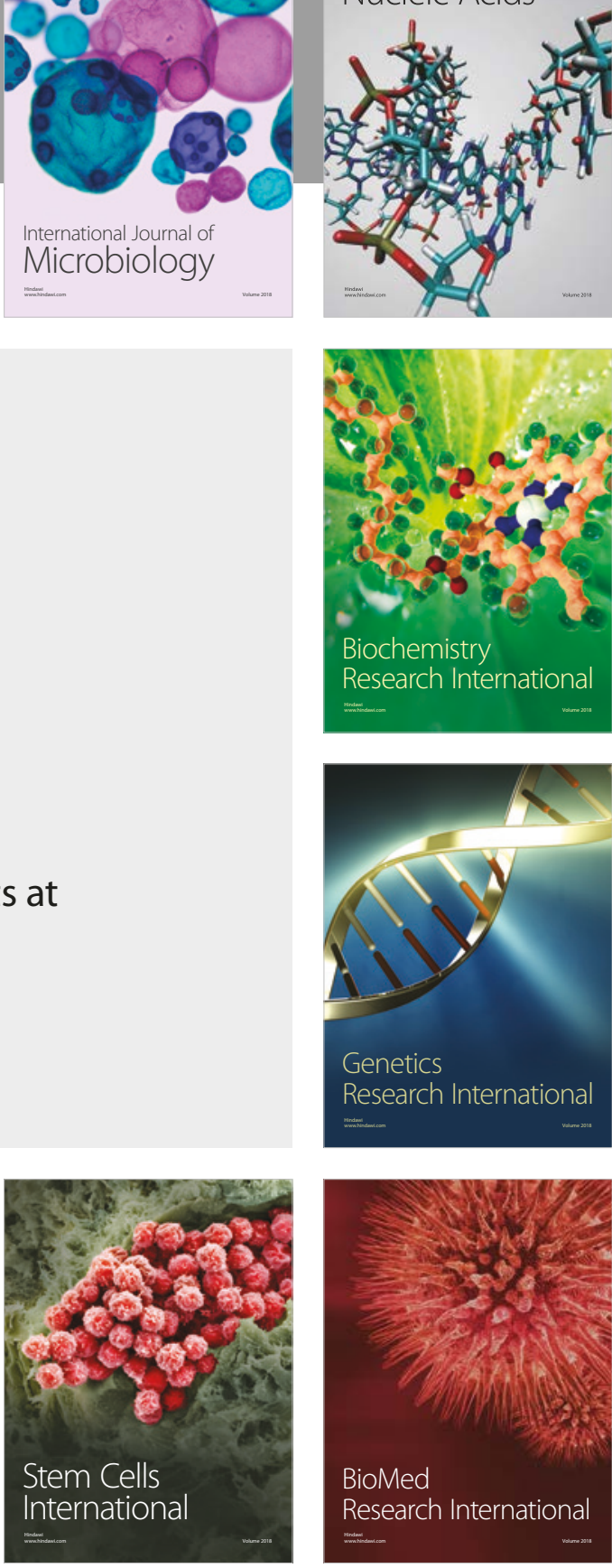
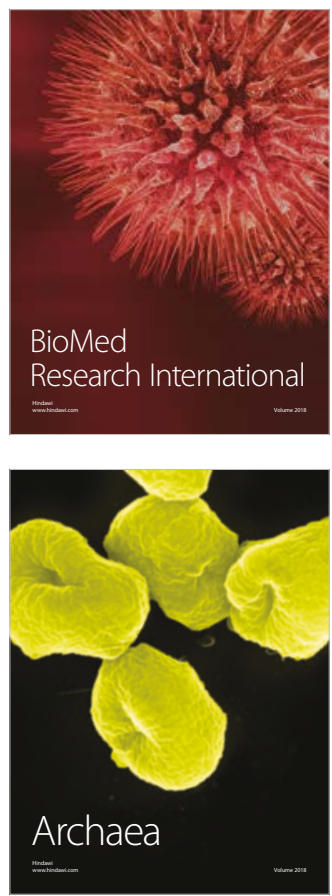